\title{
Summary of Canadian Immunization Committee (CIC) Recommendations for Human Papillomavirus Immunization Programs
}

\author{
Canadian Immunization Committee ${ }^{1 *}$
}

${ }^{*}$ Correspondence to: CIC Secretariat (cic-cci@phac-aspc.gc.ca)

\section{Background}

There are over 100 different types of human papillomavirus (HPV), and many have been linked to cancers and benign lesions of the anogenital tract, head, and neck. In women, almost all cervical cancers can be traced to infection with oncogenic HPV types, including the high-risk types 16 and 18. Of HPV-associated cancers in men, approximately $92 \%$ of anal cancers, $63 \%$ of penile cancers, and $89 \%$ of oral cavity and oropharyngeal cancers are attributable to high-risk HPV types 16 and 18. HPV-associated anogenital warts also represent a quality-of-life and an economic burden for both males and females. Publicly funded HPV immunization programs for girls are currently in place in all provinces and territories; however, uptake ranges from $60 \%$ to $85 \%$. On the basis of scientific evidence, the National Advisory Committee on Immunization (NACl) now recommends HPV immunization for males and females between 9 and 26 years of age for the prevention of HPV disease. In response to expanded indications for the quadrivalent HPV vaccine, as well as with the introduction of a new bivalent HPV vaccine, the Canadian Immunization Committee $(\mathrm{CIC})$ considered the programmatic issues of the HPV vaccines and their appropriateness for inclusion in provincial and territorial routine immunization schedules. This document is a brief summary of the full CIC HPV recommendations (1).

\section{Objective}

To make recommendations for HPV immunization programs in light of an expanded HPV immunization program goal of reducing vaccine preventable HPV-related morbidity and mortality in the Canadian population.

\section{Approach}

A Task Group of the CIC considered the following: disease characteristics and burden of illness, vaccine safety and efficacy, feasibility and acceptability, cost-effectiveness, the ability to evaluate HPV immunization programs, research questions, and equity and ethical considerations.

\section{Findings}

There are currently two HPV vaccines approved for use in Canada: Gardasil ${ }^{\circledR}$ and Cervarix $^{\mathrm{TM}}$. Gardasil ${ }^{\circledR}$, a quadrivalent vaccine, has been approved for females aged 9 to 45 years and males aged 9 to 26 years for the prevention of infection caused by HPV types $6,11,16$, and 18 . Cervarix ${ }^{\mathrm{TM}}$, a bivalent vaccine, has been approved for females aged 9 to 25 years for the prevention of cervical cancer caused by oncogenic HPV types 16 and 18. The immunogenicity, efficacy, and safety of both vaccines have been clearly demonstrated; however, some studies indicate higher immunogenicity and cross-protective vaccine efficacy for Cervarix ${ }^{\mathrm{TM}}$. The quadrivalent vaccine's protection against anogenital warts has been shown to be economically more important than the possible advantages of the bivalent vaccine in cancer prevention; thus the quadrivalent vaccine is more costeffective than the bivalent vaccine.

When considering the inclusion of males in HPV immunization programs in Canada, cost-effectiveness studies show that a male program is predicted to be cost-effective only when immunization coverage is lower than $50 \%$ among girls. If a male program targeted at high-risk boys and men (e.g. men who have sex with men) were initiated, the quadrivalent vaccine would be the product of choice, as it is the only one currently approved in Canada for use in males. As Canada's provinces and territories already have HPV immunization programs in 
place for girls, some evaluations have been published. More data is required before an evaluation of the new HPV immunization programs can occur at the national level.

Many factors must be considered when evaluating HPV immunization programs, such as the availability of systems to 1) measure coverage and vaccine utilization, and the quality of immunization services; 2) measure the impact of HPV-related infections; and 3) link health outcomes databases, immunization registries, and population registries. Research priorities for HPV immunization have been identified by the 2005 National HPV Research Priorities Workshop, the 2012 NACI statement, and the HPV Expert Group, which met in June 2013. Indicators for evaluating the impact of HPV immunization on the population are also under development. In addition to equity and ethical considerations, the impact of HPV immunization on cervical cancer screening should be considered; an immunization program should constitute part of a comprehensive cervical cancer prevention program.

\section{Recommendations}

The goal of the HPV immunization program was expanded in June 2013 from decreasing the morbidity and mortality of cervical cancer, its precursors, and other HPV-related cancers in women in Canada to reducing vaccine preventable HPV-related morbidity and mortality in the Canadian population. The expanded national goal provides flexibility for HPV immunization to consider other aspects of HPV morbidity, as well as the inclusion of males and other population subgroups in HPV immunization programs. It also continues to allow for an emphasis on the reduction of morbidity and mortality of cervical cancer, its precursors, and other HPV-related cancers in women.

In support of the new national goal, the CIC makes the following recommendations: 1) improve national coverage rates among immunization program recipients; 2) prioritize evaluation and the setting of program indicators; 3 ) address new and unresolved research priorities; and 4) integrate new population groups into immunization programs with a thoughtful, risk-based approach.

\section{Reference}

(1) Canadian Immunization Committee. Recommendations for Human Papillomavirus Immunization Programs. 2013; http://publications.gc.ca/site/eng/464264/publication.html

${ }^{1}$ Note: The Canadian Immunization Committee provides operational and technical advice related to immunization policies and programs in Canada. CIC consists of representatives from provincial and territorial jurisdictions, the National Advisory Committee on Immunization, the American Advisory Committee on Immunization Practices, and Health Canada's Biologics and Genetics Therapies Directorate, Marketed Health Products Directorate, and First Nations and Inuit Health Branch. 